\title{
Erratum to: Logarithm laws for flows on homogeneous spaces
}

\author{
D. Y. Kleinbock ${ }^{1}$ - G. A. Margulis ${ }^{2}$
}

Received: 30 June 2017 / Accepted: 20 July 2017 / Published online: 29 August 2017

(C) Springer-Verlag GmbH Germany 2017

\begin{abstract}
One of the propositions in the paper (Kleinbock and Margulis in Invent Math 138(3):451-494, 1999), related to approximating certain sets by smooth functions, was recently found to be incorrect. Here, we correct the mistake.
\end{abstract}

\section{Erratum to: Invent. math. 138, 451-494 (1999) DOI 10.1007/s002229900012}

\section{Statement of results}

Let us reproduce the setting of [3] in a slightly more general form. Let $G$ be a Lie group and $\Gamma$ a lattice in $G$. Denote by $X$ the homogeneous space $G / \Gamma$ and by $\mu$ the $G$-invariant probability measure on $X$. In what follows, $\|\cdot\|_{p}$ will stand for the $L^{p}$ norm. Fix a basis $\left\{Y_{1}, \ldots, Y_{n}\right\}$ for the Lie algebra $\mathfrak{g}$ of

The work of the first named author was supported in part by NSF Grant DMS-1600814, and that of the second named author by NSF Grant DMS-1265695.

The online version of the original article can be found under doi:10.1007/s002229900012.

$凶$ D. Y. Kleinbock

kleinboc@brandeis.edu

G. A. Margulis

grigorii.margulis@yale.edu

1 Brandeis University, Waltham, MA 02454-9110, USA

2 Department of Mathematics, Yale University, New Haven, CT 06520, USA 
$G$, and, given a smooth function $h \in C^{\infty}(X)$ and $\ell \in \mathbb{N}$, define the " $L^{2}$, order $\ell$ " Sobolev norm $\|h\|_{2, \ell}$ of $h$ by

$$
\|h\|_{2, \ell} \stackrel{\text { def }}{=} \sum_{|\alpha| \leq \ell}\left\|D^{\alpha} h\right\|_{2}
$$

where $\alpha=\left(\alpha_{1}, \ldots, \alpha_{n}\right)$ is a multiindex, $|\alpha|=\sum_{i=1}^{n} \alpha_{i}$, and $D^{\alpha}$ is a differential operator of order $|\alpha|$ which is a monomial in $Y_{1}, \ldots, Y_{n}$, namely $D^{\alpha}=Y_{1}^{\alpha_{1}} \cdots Y_{n}^{\alpha_{n}}$. This definition depends on the basis, however, a change of basis would only distort $\|h\|_{2, \ell}$ by a bounded factor. We also let

$$
C_{2}^{\infty}(X)=\left\{h \in C^{\infty}(X):\|h\|_{2, \ell}<\infty \text { for any } \ell=\mathbb{Z}_{+}\right\} .
$$

Now, let $\Delta$ be a real-valued function on $X$, and for $z \in \mathbb{R}$ denote

$$
\Phi_{\Delta}(z) \stackrel{\text { def }}{=} \mu\left(\Delta^{-1}([z, \infty))\right) .
$$

Say that $\Delta$ is $D L$ (an abbreviation for "distance-like") if there exists $z_{0} \in \mathbb{R}$ such that $\Phi_{\Delta}\left(z_{0}\right)>0$ and

(a) $\Delta$ is uniformly continuous on $\Delta^{-1}\left(\left[z_{0}, \infty\right)\right)$; that is, $\forall \varepsilon>0$ there exists a neighborhood $U$ of the identity in $G$ such that for any $x \in X$ with $\Delta(x) \geq z_{0}$,

$$
g \in U \Longrightarrow|\Delta(x)-\Delta(g x)|<\varepsilon
$$

(b) the function $\Phi_{\Delta}$ does not decrease very fast, more precisely, if

$$
\exists c, \delta>0 \text { such that } \Phi_{\Delta}(z) \geq c \Phi_{\Delta}(z-\delta) \quad \forall z \geq z_{0} .
$$

The paper [3] gives several examples of DL functions on homogeneous spaces of semisimple Lie groups. The main goal of that paper was to study statistics of excursions of generic trajectories of flows on $X$ into sets $\Delta^{-1}([z, \infty))$ for large enough $z$. A crucial ingredient of the argument was approximation of characteristic functions of those sets by smooth functions with uniformly bounded Sobolev norms. However, as was recently observed by Dubi Kelmer and Shucheng Yu, the argument in the main approximation statement, namely [3, Lemma 4.2], contains a mistake. To state a corrected version below, we need to weaken the regularity assumption on the smooth functions approximating the sets $\Delta^{-1}([z, \infty))$. Namely, for $\ell \in \mathbb{Z}_{+}$and $C>0$, let us say that a nonnegative function $h \in C_{2}^{\infty}(X)$ is $(C, \ell)$-regular if

$$
\|h\|_{2, \ell} \leq C \sqrt{\|h\|_{1}} .
$$


Note that the argument of [3] used a stronger condition:

$$
\|h\|_{2, \ell} \leq C\|h\|_{1} .
$$

(REG-old)

Equivalently one can replace $\sqrt{\|h\|_{1}}$ in (REG) with $\|h\|_{2}$, but for technical reasons it is more convenient to use the square root of the $L^{1}$ norm. Here is the corrected statement of [3, Lemma 4.2]:

Theorem 1.1 Let $\Delta$ be a DLfunction on $X$. Then, for any $\ell \in \mathbb{Z}_{+}$there exists $C>0$ such that for every $z \geq z_{0}$ one can find two $(C, \ell)$-regular nonnegative functions $h^{\prime}$ and $h^{\prime \prime}$ on $X$ such that

$$
h^{\prime} \leq 1_{\Delta^{-1}([z, \infty))} \leq h^{\prime \prime} \text { and } c \Phi_{\Delta}(z) \leq\left\|h^{\prime}\right\|_{1} \leq\left\|h^{\prime \prime}\right\|_{1} \leq \frac{1}{c} \Phi_{\Delta}(z),
$$

with $c$ and $z_{0}$ as in (DL).

Fix a right-invariant Riemannian metric on $G$ and the corresponding metric 'dist' on $X$. For $g \in G$, let us denote by $\|g\|$ the distance between $g \in G$ and the identity element of $G$. (Note that $\|g\|=\left\|g^{-1}\right\|$ due to the right invariance of the metric.) Now say that the $G$-action on $X$ is exponentially mixing if there exist $\lambda, E>0$ and $\ell \in \mathbb{Z}_{+}$such that for any $\varphi, \psi \in C_{2}^{\infty}(X)$ and for any $g \in G$ one has

$$
|\langle g \varphi, \psi\rangle| \leq E e^{-\lambda\|g\|}\|\varphi\|_{2, \ell}\|\psi\|_{2, \ell} .
$$

Here $\langle\cdot, \cdot\rangle$ stands for the inner product in $L^{2}(X, \mu)$.

One of the main goals of [3] was given a sequence $\left\{f_{t}: t \in \mathbb{N}\right\}$ of elements of $G$ and a sequence of nonnegative functions $\left\{h_{t}: t \in \mathbb{N}\right\}$ on $X$ such that

$$
\sum_{t=1}^{\infty}\left\|h_{t}\right\|_{1}=\infty
$$

to compare the growth of $\sum_{t=1}^{N} h_{t}\left(f_{t} x\right)$ for $\mu$-a.e. $x \in X$ with the growth of $\sum_{t=1}^{N}\left\|h_{t}\right\|_{1}$ as $N \rightarrow \infty$. Results like that usually go by the name 'dynamical Borel-Cantelli lemmas,' see [1,2]. In [3, Proposition 4.1], such a conclusion was shown to follow from the exponential mixing of the $G$-action on $X$, the exponential divergence of $\left\{f_{t}\right\}$, namely the condition

$$
\sup _{t \in \mathbb{N}} \sum_{s=1}^{\infty} e^{-\lambda\left\|f_{s} f_{t}^{-1}\right\|}<\infty \quad \forall \lambda>0,
$$

and the regularity assumption (REG-old) on functions $\left\{h_{t}\right\}$. 
In the following theorem, we weaken the regularity condition (REG-old) to (REG) and derive the same conclusion:

Theorem 1.2 Suppose that the $G$-action on $X$ is exponentially mixing. Let $\left\{f_{t}: t \in \mathbb{N}\right\}$ be a sequence of elements of $G$ satisfying (ED), and let $\left\{h_{t}\right.$ : $t \in \mathbb{N}\}$ be a sequence of nonnegative $(C, \ell)$-regular functions on $X$ such that $\left\|h_{t}\right\|_{1} \leq 1$ for all $t$, and $\sum_{t=1}^{\infty}\left\|h_{t}\right\|_{1}=\infty$. Then

$$
\lim _{N \rightarrow \infty} \frac{\sum_{t=1}^{N} h_{t}\left(f_{t} x\right)}{\sum_{t=1}^{N}\left\|h_{t}\right\|_{1}}=1 \text { for } \mu \text {-a.e. } x \in X .
$$

Using the above theorem in place of [3, Proposition 4.1] and Theorem 1.1 in place of [3, Lemma 4.2], one can then recover [3, Theorem 4.3], that is, prove

Theorem 1.3 Suppose that the $G$-action on $X$ is exponentially mixing. Let $\left\{f_{t}: t \in \mathbb{N}\right\}$ be a sequence of elements of $G$ satisfying (ED), let $\Delta$ be a $D L$ function on $X$, and let $\left\{r_{t}: t \in \mathbb{N}\right\} \subset\left[z_{0}, \infty\right)$ be such that

$$
\sum_{t=1}^{\infty} \Phi_{\Delta}\left(r_{t}\right)=\infty
$$

Then, for some positive $c \leq 1$ and for almost all $x \in X$ one has

$$
\begin{aligned}
c & \leq \liminf _{N \rightarrow \infty} \frac{\#\left\{1 \leq t \leq N \mid \Delta\left(f_{t} x\right) \geq r_{t}\right\}}{\sum_{t=1}^{N} \Phi_{\Delta}\left(r_{t}\right)} \\
& \leq \limsup _{N \rightarrow \infty} \frac{\#\left\{1 \leq t \leq N \mid \Delta\left(f_{t} x\right) \geq r_{t}\right\}}{\sum_{t=1}^{N} \Phi_{\Delta}\left(r_{t}\right)} \leq \frac{1}{c} .
\end{aligned}
$$

Consequently, for any $\left\{r_{t}\right\}$ satisfying (1.2) and almost all $x \in X$ one has $\Delta\left(f_{t} x\right) \geq r_{t}$ for infinitely many $t \in \mathbb{N}$. That is, in the terminology of [3], the family of sets

$$
\left\{\Delta^{-1}([z, \infty)): z \in \mathbb{R}\right\}
$$

is Borel-Cantelli for $\left\{f_{t}\right\}$.

\section{Proofs}

Let us state a general form of Young's inequality, whose proof we give for the sake of self-containment of the paper. Denote by $m$ the Haar measure on 
$G$ normalized so that the quotient map $G \rightarrow X$ locally sends $m$ to $\mu$. For $\psi \in L^{1}(G, m)$ and $h \in L^{1}(X, \mu)$, define $\psi * h$ by

$$
(\psi * h)(x) \stackrel{\text { def }}{=} \int_{G} \psi(g) h\left(g^{-1} x\right) d m(g) .
$$

Lemma 2.1 Let $\psi \in L^{1}(G, m)$ and $h \in L^{2}(X, \mu)$. Then $\|\psi * h\|_{2} \leq$ $\|\psi\|_{1}\|h\|_{2}$.

Proof We have

$$
\begin{aligned}
|(\psi * h)(x)| \leq & \int_{G}|\psi(g)|^{1 / 2}\left|h\left(g^{-1} x\right)\right| \cdot|\psi(g)|^{1 / 2} d m(g) \\
\text { (by Cauchy-Schwarz) } \leq & \left(\int_{G}|\psi(g)| d m(g)\right)^{1 / 2} \\
& \times\left(\int_{G}\left|h\left(g^{-1} x\right)\right|^{2}|\psi(g)| d m(g)\right)^{1 / 2} .
\end{aligned}
$$

Thus, we have

$$
|(\psi * h)(x)|^{2} \leq\|\psi\|_{1} \int_{G}|\psi(g)| \cdot\left|h\left(g^{-1} x\right)\right|^{2} d m(g) .
$$

Integrating over $X$ and using Fubini's Theorem gives

$$
\begin{aligned}
\|\psi * h\|_{2}^{2} & \leq\|\psi\|_{1} \int_{X} \int_{G}|\psi(g)| \cdot\left|h\left(g^{-1} x\right)\right|^{2} d m(g) d \mu(x) \\
& =\|\psi\|_{1} \int_{G}|\psi(g)| \int_{X}\left|h\left(g^{-1} x\right)\right|^{2} d \mu(x) d m(g),
\end{aligned}
$$

which, by the $G$-invariance of $\mu$, is the same as

$\|\psi\|_{1} \int_{G}|\psi(g)| d m(g) \int_{X}|h(x)|^{2} d \mu(x)=\|\psi\|_{1}^{2} \cdot\|h\|_{2}^{2}$.

Proof of Theorem 1.1 We follow the proof of [3, Lemma 4.2]. For $z \in \mathbb{R}$, let us use the notation

$$
A(z) \stackrel{\text { def }}{=} \Delta^{-1}([z, \infty)) \text {. }
$$


Then, for $\varepsilon>0$, let us denote by $A^{\prime}(z, \varepsilon)$ the set of all points of $A(z)$ which are not $\varepsilon$-close to $\partial A(z)$, i.e.

$$
A^{\prime}(z, \varepsilon) \stackrel{\text { def }}{=}\{x \in A(z): \operatorname{dist}(x, \partial A(z)) \geq \varepsilon\},
$$

and by $A^{\prime \prime}(z, \varepsilon)$ the $\varepsilon$-neighborhood of $A(z)$, namely

$$
A^{\prime \prime}(z, \varepsilon) \stackrel{\text { def }}{=}\{x \in X: \operatorname{dist}(x, A(z)) \leq \varepsilon\}
$$

Choose $z_{0}, \delta$ and $c$ as in (DL). Then, using the uniform continuity of $\Delta$ on $\Delta^{-1}\left(\left[z_{0}, \infty\right)\right)$, find $\varepsilon>0$ such that

$$
|\Delta(x)-\Delta(y)|<\delta \text { whenever } \Delta(x) \geq z_{0} \text { and } \operatorname{dist}(x, y)<\varepsilon .
$$

It follows that for all $z \geq z_{0}$,

$$
A(z+\delta) \subset A^{\prime}(z, \varepsilon) \subset A(z) \subset A^{\prime \prime}(z, \varepsilon) \subset A(z-\delta) ;
$$

therefore, one can apply (DL) to conclude that

$$
c \mu(A(z)) \leq \mu\left(A^{\prime}(z, \varepsilon)\right) \leq \mu\left(A^{\prime \prime}(z, \varepsilon)\right) \leq \frac{1}{c} \mu(A(z)) .
$$

Now, take a nonnegative $\psi \in C^{\infty}(G)$ of $L^{1}$ norm 1 such that supp $\psi$ belongs to the ball of radius $\varepsilon / 4$ centered in $e \in G$. Fix $z \geq z_{0}$ and consider functions $h^{\prime} \stackrel{\text { def }}{=} \psi * 1_{A^{\prime}(z, \varepsilon / 2)}$ and $h^{\prime \prime} \stackrel{\text { def }}{=} \psi * 1_{A^{\prime \prime}(z, \varepsilon / 2)}$. Then, one clearly has

$$
1_{A^{\prime}(z, \varepsilon)} \leq h^{\prime} \leq 1_{A(z)} \leq h^{\prime \prime} \leq 1_{A^{\prime \prime}(z, \varepsilon)}
$$

which, together with (2.1), immediately implies (1.1). It remains to choose $\ell \in \mathbb{Z}_{+}$and find $C$ (independent of $z$ ) such that both $h^{\prime}$ and $h^{\prime \prime}$ are $(C, \ell)$ regular. Take a multiindex $\alpha$ with $|\alpha| \leq \ell$, and write

$$
\left\|D^{\alpha} h^{\prime}\right\|_{2}=\left\|D^{\alpha}\left(\psi * 1_{A^{\prime}(z, \varepsilon / 2)}\right)\right\|_{2}=\left\|D^{\alpha}(\psi) * 1_{A^{\prime}(z, \varepsilon / 2)}\right\|_{2} .
$$

Then, by the Young inequality,

$$
\begin{aligned}
& \left\|D^{\alpha} h^{\prime}\right\|_{2} \leq\left\|D^{\alpha}(\psi)\right\|_{1} \sqrt{\mu\left(A^{\prime}(z, \varepsilon / 2)\right)} \leq\left\|D^{\alpha}(\psi)\right\|_{1} \sqrt{\mu(A(z))} \\
& \leq\left\|D^{\alpha}(\psi)\right\|_{1}\left(\frac{\left\|h^{\prime}\right\|_{1}}{c}\right)^{1 / 2} .
\end{aligned}
$$


Similarly,

$$
\begin{aligned}
& \left\|D^{\alpha} h^{\prime \prime}\right\|_{2} \leq\left\|D^{\alpha}(\psi)\right\|_{1} \sqrt{\mu\left(A^{\prime \prime}(z, \varepsilon / 2)\right)} \leq\left\|D^{\alpha}(\psi)\right\|_{1}\left(\frac{\mu(A(z))}{c}\right)^{1 / 2} \\
& \leq\left\|D^{\alpha}(\psi)\right\|_{1}\left(\frac{\left\|h^{\prime \prime}\right\|_{1}}{c}\right)^{1 / 2} ;
\end{aligned}
$$

hence, with $C=\frac{1}{\sqrt{c}} \sum_{|\alpha| \leq \ell}\left\|D^{\alpha}(\psi)\right\|_{1}$, both $h^{\prime}$ and $h^{\prime \prime}$ are $(C, \ell)$-regular, and the theorem is proven.

Proof of Theorem 1.2 Denote $\int_{X} h_{t} d \mu=\left\|h_{t}\right\|_{1}$ by $a_{t}$. Following the argument in [3], our goal is to show that the sequence of functions $\left\{h_{t} \circ f_{t}\right\}$ satisfies a second-moment condition dating back to the work of Schmidt and Sprindžuk:

$$
\sup _{1 \leq M<N} \frac{\int_{X}\left(\sum_{t=M}^{N} h_{t}\left(f_{t} x\right)-\sum_{t=M}^{N} a_{t}\right)^{2} d \mu}{\sum_{t=M}^{N} a_{t}}<\infty .
$$

the conclusion of the theorem will then follow in view of [3, Lemma 2.6], which is a special case of [4, Chapter I, Lemma 10].

Take $1 \leq M<N$. As in [3, Remark 2.7], one can rewrite the numerator as $\sum_{s, t=M}^{N}\left(\left\langle f_{t}^{-1} h_{t}, f_{s}^{-1} h_{s}\right\rangle-a_{s} a_{t}\right)$ and then estimate it using the exponential mixing of the $G$-action on $X$ :

$$
\begin{aligned}
\left|\sum_{s, t=M}^{N}\left\langle f_{t}^{-1} h_{t}, f_{s}^{-1} h_{s}\right\rangle-a_{s} a_{t}\right| & \leq \sum_{s, t=M}^{N}\left|\left\langle f_{s} f_{t}^{-1} h_{t}, h_{s}\right\rangle-a_{s} a_{t}\right| \\
(\text { with } E, \lambda, \ell \text { as in (EM) }) & \leq E \sum_{s, t=M}^{N} e^{-\lambda\left\|f_{s} f_{t}^{-1}\right\|}\left\|h_{t}\right\|_{2, \ell}\left\|h_{s}\right\|_{2, \ell}
\end{aligned}
$$

(by the $(C, \ell)$-regularity of $\left.\left\{h_{t}\right\}\right) \leq E C^{2} \sum_{s, t=M}^{N} e^{-\lambda\left\|f_{s} f_{t}^{-1}\right\|} \sqrt{a_{s} a_{t}}$.

Now, following an observation communicated to us by Shucheng Yu, split the above sum according to the comparison between $a_{s}$ and $a_{t}$ :

$$
\sum_{a_{s}=a_{t}} e^{-\lambda\left\|f_{s} f_{t}^{-1}\right\|} \sqrt{a_{s} a_{t}}+\sum_{a_{s}<a_{t}} e^{-\lambda\left\|f_{s} f_{t}^{-1}\right\|} \sqrt{a_{s} a_{t}}+\sum_{a_{s}>a_{t}} e^{-\lambda\left\|f_{s} f_{t}^{-1}\right\|} \sqrt{a_{s} a_{t}},
$$


where the values of $s, t$ in the last three sums range between $M$ and $N$. By symmetry, the last two sums are equal. Thus, (2.2) is not greater than

$$
\begin{aligned}
\sum_{a_{s}=a_{t}} e^{-\lambda\left\|f_{s} f_{t}^{-1}\right\|} a_{t}+2 \sum_{a_{s}<a_{t}} e^{-\lambda\left\|f_{s} f_{t}^{-1}\right\|} a_{t} & \leq 2 \sum_{s, t=M}^{N} e^{-\lambda\left\|f_{s} f_{t}^{-1}\right\|} a_{t} \\
& \leq 2 \sum_{t=M}^{N} a_{t} \sum_{s=M}^{N} e^{-\lambda\left\|f_{s} f_{t}^{-1}\right\|} \\
& \leq 2 \sum_{t=M}^{N} a_{t} \cdot \sup _{t \in \mathbb{N}} \sum_{s=1}^{\infty} e^{-\lambda\left\|f_{s} f_{t}^{-1}\right\|},
\end{aligned}
$$

and the proof of (SP) is finished in view of (ED).

Acknowledgements The authors are grateful to Dubi Kelmer for bringing their attention to the mistake in [3, Lemma 4.2], and to Shucheng Yu for a suggestion how to correct it. Thanks are also due to Nick Wadleigh for helpful discussions.

\section{References}

1. Chernov, N., Kleinbock, D.: Dynamical Borel-Cantelli lemmas for Gibbs measures. Isr. J. Math. 122, 1-27 (2001)

2. Haydn, N., Nicol, M., Persson, T., Vaienti, S.: A note on Borel-Cantelli lemmas for nonuniformly hyperbolic dynamical systems. Ergod. Theory Dyn. Syst. 33(2), 475-498 (2013)

3. Kleinbock, D., Margulis, G.A.: Logarithm laws for flows on homogeneous spaces. Invent. Math. 138(3), 451-494 (1999)

4. Sprindžuk, V.: Metric Theory of Diophantine Approximations. Wiley, New York (1979) 